\title{
BMJ Open East Midlands knee pain multiple randomised controlled trial cohort study: cohort establishment and feasibility study protocol
}

\author{
Michelle Hall (D) ,,2 Amy Fuller, ${ }^{2,3}$ Polykarpos Angelos Nomikos, ${ }^{2,3}$ Bonnie Millar, ${ }^{2,4}$ \\ Reuben Ogollah, ${ }^{5}$ Ana Valdes, ${ }^{2,4}$ Paul Greenhaff, ${ }^{2,6}$ Roshan das Nair, ${ }^{7,8}$ \\ Michael Doherty, ${ }^{3,4}$ David A Walsh, ${ }^{2,4}$ A Abhishek ${ }^{2,3}$
}

To cite: Hall M, Fuller A, Nomikos PA, et al. East Midlands knee pain multiple randomised controlled trial cohort study: cohort establishment and feasibility study protocol. BMJ Open 2020;10:e037760. doi:10.1136/ bmjopen-2020-037760

- Prepublication history for this paper is available online. To view these files, please visit the journal online (http://dx.doi org/10.1136/bmjopen-2020037760).

Received 18 February 2020 Revised 11 June 2020 Accepted 22 July 2020

Check for updates

(C) Author(s) (or their employer(s)) 2020. Re-use permitted under CC BY-NC. No commercial re-use. See rights and permissions. Published by BMJ.

For numbered affiliations see end of article.

Correspondence to

Dr Michelle Hall;

michelle.hall@nottingham.ac.uk

\section{ABSTRACT}

Introduction Knee pain due to osteoarthritis $(O A)$ is a common cause of disability. The UK National Institute for Health and Care Excellence OA guidelines recommend education, exercise and weight loss advice (if overweight) as core interventions before pharmacological adjuncts. However, implementation of these in primary care is often suboptimal. This study aims to develop a complex intervention with non-pharmacological and pharmacological components that can be delivered by nurses. The feasibility and acceptability of the intervention, and feasibility of undertaking a future cohort randomised controlled trial (RCT) will be explored.

Methods and analysis In phase 1, we will develop a training programme for nurses and evaluate the fidelity and acceptability of the non-pharmacological element of the intervention. Fidelity checklists completed by the nurse will be compared with video analysis of the treatment sessions. Patients and nurses will be interviewed to determine the acceptability of the intervention and explore challenges to intervention delivery. The nonpharmacological component will be modified based on the findings. In phase 2, we will assess the feasibility of conducting a cohort RCT comprising both the pharmacological and modified non-pharmacological components. We will compare three groups: group A will receive the non-pharmacological components delivered before pharmacological components; group B will receive pharmacological components followed by the nonpharmacological components; and group C (control arm) will continue to receive usual care. Study outcomes will be collected at three time points: baseline, 13 and 26 weeks after randomisation. Qualitative interviews will be conducted with a sample of participants from each of the two active intervention arms.

Ethics and dissemination This protocol was approved by the East Midlands-Derby Research Ethics Committee (18/EM/0288) and registered at ClinicalTrials.gov (protocol v4.0, 10/02/2020). The study will be reported in accordance with the Consolidated Standards of Reporting Trials guidance and standards. The results will be submitted for publication in peer-reviewed academic journals.

Trial registration number NCT03670706

\section{Strengths and limitations of this study}

- First study to develop and evaluate the feasibility of a wholly nurse-led intervention following the core National Institute for Health and Care Excellence guidelines for treating knee pain and osteoarthritis (OA).

- This study will not determine the effectiveness of this model of care for knee pain and $O A$, but will explore the feasibility of implementation and running an adequately powered randomised controlled trial (RCT) and determine signal of efficacy.

- Assessing fidelity of intervention delivery will allow us to explore the extent to which the nurses can deliver individual components of the complex package of care as planned, and alongside exploring its acceptability will inform refinements to nurse training and/or the package of care.

- Blinded outcome assessment.

- Participants and nurses delivering care will not be blinded to each intervention. However, in using a cohort RCT study design where participants will be informed that their data may be used as a control comparison for intervention studies, bias associated with disappointment, behaviour modification and dropouts in the control arm will be minimised.

\section{INTRODUCTION}

Chronic knee pain affects one in four people aged 55 years or older, and is most commonly caused by osteoarthritis (OA). ${ }^{1}$ The global prevalence of symptomatic knee OA is estimated at $4 \%$, and it has an important impact on both affected individuals and health and social care systems. ${ }^{2}{ }^{3}$ The socioeconomic and healthcare burden of knee OA is likely to increase due to the ageing population and the obesity epidemic. ${ }^{24}$

Best practice guidelines for managing OA published by the National Institute for Health and Care Excellence (NICE) recommend individualised patient education, advice 
and access to information about $\mathrm{OA}$ and its management, strengthening and aerobic exercise, and guidance on losing weight if applicable as core treatments, with consideration of adjunctive pharmacological and other non-pharmacological treatments as required. ${ }^{5}$ However, core treatments are frequently underused as both doctors and patients predominantly focus on pharmacological options including opioids. ${ }^{67}$ Most people with OA feel they do not receive the treatment they need from general practitioners (GP), with an overemphasis on prescription drugs and lack of discussion about exercise and diet. ${ }^{8}$ Given the growing concerns about the overuse of opioids for musculoskeletal pain including $\mathrm{OA},{ }^{9}$ it is important to explore alternative models of healthcare delivery with an emphasis on non-pharmacological interventions for this condition. Additionally, while it has been suggested that optimising analgesia prior to participation in exercise therapy may enhance adherence and patient outcomes, this has not been confirmed in a randomised controlled trial (RCT). ${ }^{10}$

Alternative models for implementing OA care have shown potential. The MOSAICS trial explored the effectiveness of delivering an enhanced initial consultation with GP and provision of nurse-led follow-ups as a clinically practicable way of implementing NICE guidelines compared with usual care. ${ }^{11}$ However, only $29 \%$ of patients in the intervention arm reported having a consultation with a nurse, making it difficult to determine whether a nurse could help deliver the core NICE recommendations. Others have included using community physiotherapists and pharmacists for delivering interventions but have only demonstrated short-term improvements in health outcomes. ${ }^{12}$

The majority of patients with knee pain self-manage their symptoms, and those who seek healthcare are managed by their GPs and community physiotherapists in the first instance. ${ }^{8}$ A potential role for practice nurses has been identified ${ }^{13}$ and nurse-led clinics already exist for patients with long-term conditions such as coronary heart disease,${ }^{14}$ heart failure ${ }^{15}$ and diabetes ${ }^{16}$ resulting in equivalent or better outcomes for patients compared with usual GP-led care. Upskilling practice nurses to deliver the management of long-term conditions is recognised as a key strategy for the future of primary care. ${ }^{17}$ This paper describes the protocol for developing and testing the feasibility of a wholly nurse-led intervention for people with knee pain, delivering the core NICE recommendations.

Further to this, it will test the feasibility of a cohort RCT. The usual double-blind, placebo-controlled RCT cannot be used when investigating the efficacy of complex interventions for knee pain as it is not possible to blind participants to their treatment. This has led to the use of pragmatic RCTs where participants are randomised to receive an intervention or continue usual primary care. ${ }^{12}{ }^{18}$ Such studies can be affected by disappointment bias, behaviour modification bias and differential dropouts when those selected as controls had hoped to receive the intervention under investigation and drop out when allocated to the control arm. Cohort RCTs, as will be used in this study, have been adopted to prevent such biases. ${ }^{19}{ }^{20}$ In this study design, a cohort of people with the condition of interest are recruited and consented to be approached to complete further questionnaire surveys, for their GP medical records and prescriptions to be accessed, to be used as a comparator for future studies and to be approached for participation in future studies if eligible. ${ }^{21}$ Clinical and patient-reported outcomes are captured at regular intervals, allowing those eligible for any given study to act as a control without being informed about the experimental intervention and thus minimising the bias associated with pragmatic trials.

\section{Aims and objectives}

The overall purpose is to develop and test the feasibility of a nurse-led intervention for people with knee pain using a cohort RCT study design.

This study has two phases:

Phase 1 involves the development and evaluation of the non-pharmacological treatment component. Specific objectives of phase 1 are to: (1) develop a training package for nurses to deliver the core non-pharmacological and pharmacological principles to manage knee OA as recommended by NICE; (2) determine the fidelity of delivery of the nurse-delivered components of the intervention; (3) explore patient and nurse acceptability of the nonpharmacological components of the intervention.

Phase 2 will test the feasibility of a cohort RCT of nurse-led versus usual care of people with knee pain and explore whether such a trial should provide analgesia before non-pharmacological interventions.

\section{METHODS}

\section{Participants}

Participants eligible for both phases of the study will be aged 40 years or older and self-reporting knee pain on most days of the previous month for at least 3 months. Knee pain severity will be scored between 4 and 7 on a 0-10 numeric rating scale (NRS). This will be assessed using the following question: 'Over the past 4-weeks, how intense was the average pain or aching in your knees on a $0-10$ scale, where 0 is no pain and 10 is pain as bad as could be?'

Exclusion criteria include participants who are unable to communicate in English, who are housebound or care home residents, on dialysis or home oxygen, pregnant or have dementia, serious mental illness, terminal cancer, autoimmune rheumatic diseases, asthma or lung disease requiring regular daily oral corticosteroids, unstable angina or heart failure, known peripheral vascular disease, stroke with residual weakness or sensory loss, physician-diagnosed peripheral neuropathy with sensory or motor deficit, previous knee or hip replacement, on a waiting list for a knee or hip replacement or with severe knee pain (score $>7$ on NRS for average pain in the past 4 weeks). 


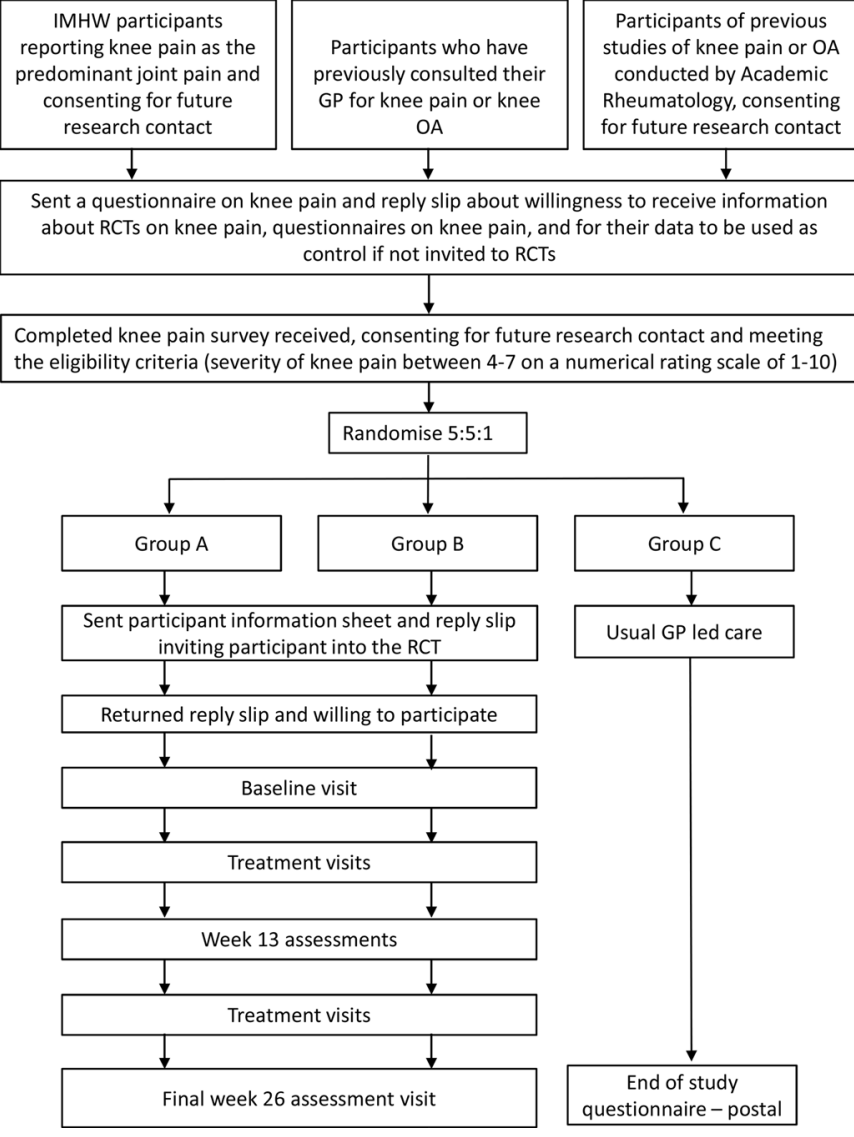

Figure 1 Participant timeline through the study. GP, general practitioner; IMHW, Investigating Musculoskeletal Health and Wellbeing cohort study; OA, osteoarthritis; RCT, randomised controlled trial.

Nurses who undergo the intervention training and delivered the intervention in the study will be recruited to the qualitative components of the study and will give their consent to be interviewed in both phases of the study.

\section{Recruitment}

There are three routes of recruitment:

1. Participants will primarily be recruited from the Investigating Musculoskeletal Health and Wellbeing survey (NCT03696134), a cohort study that aims to measure and characterise the development and progression of pain, frailty and disability and form a longitudinal context for nested research. ${ }^{21}$ Participants who self-report knee pain as their predominant body pain and consent for future research contact will be sent a questionnaire enquiring about their knee pain, mood, function and quality of life. They will also be asked about willingness to receive information about trials on knee pain, complete further questionnaires on knee pain, and for their data to be used for comparisons with other participant groups in research studies. Those willing and meeting the eligibility criteria will be invited to take part in the current study (figure 1).

2. Additional participants identified by screening of GP records for previous consultation for knee pain will be sent a questionnaire as outlined above.
3. Finally, people with knee pain or OA who have taken part in previous community-based surveys in Academic Rheumatology, University of Nottingham and consented for future research contact may be approached with a questionnaire.

\section{Setting}

Study visits for research assessment will occur at Academic Rheumatology, City Hospital Nottingham, UK and the David Greenfield Unit, Queen's Medical Centre, Nottingham, UK. The intervention will be delivered at Academic Rheumatology, City Hospital Nottingham.

\section{Consent and withdrawal}

All participants will give written informed consent before entering the study and before any assessments or interventions related to the study are undertaken. Optional consent will be sought for video recording of the intervention sessions to evaluate fidelity and audio recording of participant interviews. Participants will be free to withdraw at any time if they desire to do so, or at the discretion of the chief investigator. In the event of withdrawal, any data collected up until that point will be kept and potentially included in any analyses.

\section{Patient and public involvement}

The design of this study was supported by a patient advisory group of three people with hip or knee OA and a range of experiences in primary and secondary care. The group recognised the challenges of providing full explanations and individualised advice in a time-limited GP consultation and agreed that a wholly nurse-led intervention would be acceptable or preferable to most people. They provided input into the content of the both pharmacological and non-pharmacological components, the number and length of intervention sessions and the use of exercise diaries and educational content.

\section{PHASE 1: DEVELOPMENT OF THE NON-PHARMACOLOGICAL COMPONENT}

The non-pharmacological intervention will be developed according to the Medical Research Council framework for developing complex packages of care. ${ }^{22}$ It was informed by several strands of evidence including current guidelines, an expert multidisciplinary team of physiotherapists, rheumatologists and nurses, patient opinion, physiological considerations and behaviour change theory.

In brief, the intervention consists of a holistic assessment of the participant, individualised education about $\mathrm{OA}$, aerobic and strengthening exercises and weight loss advice if required. Evidence-based strategies to motivate participants and support adherence to healthy behaviours will be employed.

\section{The training package}

A training package for nurses will be developed and piloted during this phase. Research nurses will be recruited 
without any prior knowledge of treating musculoskeletal conditions. The content will be based on current NICE guidelines for the management of $\mathrm{OA}^{5}$ and a report by Arthritis Research UK on the educational needs of health professionals working with people with OA. ${ }^{23}$ Academic and clinical experts and members of a patient and public involvement group have provided input into the training package.

Delivery of the training package will be underpinned by educational theory ${ }^{24}$ and will build on the nurses existing knowledge of the condition. Training will be delivered in face-to-face sessions, complemented by a range of learning resources including a manual, case studies, online resources and patient simulations. Key components of the training will include:

- The epidemiology and nature of knee pain and knee OA.

- Assessment of the patient with knee OA.

- Core NICE guidelines for managing OA.

- Principles of strengthening and aerobic exercise prescription for knee OA.

- Information and advice to support weight loss.

- Strategies to support behaviour change.

- Pharmacological management of OA and knee pain following a stepwise protocol of optimising analgesia.

\section{Study design}

A pre-post intervention study using mixed quantitative and qualitative methods to determine the fidelity of delivery and the acceptability of the intervention by patients and nurses. After the nurse training, 20 participants with knee pain will be recruited to this study. They will receive the non-pharmacological components of the intervention covering as described above in four sessions over a 5-week period.

\section{Quantitative methods}

Fidelity of delivery refers to the assessment of whether content of the sessions was delivered as intended. This will be assessed using a priori fidelity checklist of five to eight categories and individual components (online supplementary additional file 1). Categories included materials, introduction, assessment, education, exercise, weight loss, adjunct treatments, and review and planning. All interventions in this phase will be video recorded (with consent). Nurses will self-complete the checklist after each session and a researcher will independently complete the checklist using the video recordings. The individual components of each category for each session will be rated as being complete, partially complete, not completed or not applicable. A sample of video recordings will be reviewed by a second researcher to determine inter-rater reliability.

\section{Quantitative analysis}

Fidelity scores will be presented as the percentage of components that were delivered as intended for the overall delivery of the intervention, for each session and for each category. Inter-rater reliability between the two researchers scoring the video recordings will be reported, as will the level of agreement between nurse-completed scores and researcher-completed (video) scores. For delivery of complex interventions such as this, levels of fidelity have been previously interpreted as 'high' fidelity where $80 \%-100 \%$ of the specified components were delivered as intended, 'moderate' $51 \%-79 \%$ and 'low' $0 \%-50 \% .{ }^{25}$ Where the fidelity scores are less than $80 \%$, we will explore further to establish which components are responsible.

\section{Qualitative methods}

Acceptability of the intervention will be explored in a face-to-face interview with all participants who received the intervention. Participants who withdraw from the intervention will be offered the opportunity to take part in an interview to explore their experiences and reasons for discontinuation. The nurses who deliver the intervention will also be invited to interview, to explore their views on the training, experience in delivering the intervention and perceived factors influencing the fidelity of delivery. Interviews will be carried out by a PhD student (PAN) and overseen by two experienced qualitative researchers $(\mathrm{AF}$ and $\mathrm{RdN}$ ). All interviews will be audio recorded and transcribed verbatim by an external specialist company Transcribe It and anonymised.

\section{Qualitative analysis}

After data transcription, all data will be checked for accuracy before transcripts are imported to NVivo V.12. Qualitative data will be analysed using a framework approach. ${ }^{26}$ This method sits within the broad family of thematic analysis, but is particularly useful for research that has specific questions and a priori issues that need to be dealt with. ${ }^{27}$ The analysis will follow the five stages of framework analysis: familiarisation with the data, construction of an initial thematic framework, indexing and sorting the data using initial thematic framework, finalisation of thematic framework, and summarising and displaying the data into a matrix. Emergent themes and subthemes will be discussed and agreed by at least two researchers to increase the validity of the analyses.

Following the fidelity evaluation and qualitative interviews, modifications may be made to study materials, procedures or protocol, and/or nurse training.

\section{PHASE 2: FEASIBILITY COHORT RCT \\ Trial design}

This will be a single-centre, mixed methods feasibility cohort RCT. Participants will be recruited as described above and randomised to one of three treatment arms (figure 1).

Group A will receive the non-pharmacological protocol for 13 weeks followed by the pharmacological protocol between weeks 13 and 26 as required. 
Group B will receive the pharmacological protocol in the first 13 weeks followed by the non-pharmacological protocol between weeks 13 and 26 with optimised background analgesia.

Group C is a control (cohort) group and will continue to receive usual care.

\section{Randomisation and allocation concealment}

Participants will be individually randomised on a 5:5:1 ratio using randomly permuted block sizes of 3 and 6 , stratified for the number of eligible knees (ie, unilateral or bilateral knee pain). Randomisation codes will be generated by the study statistician. Allocations to groups will be enclosed in serially numbered, opaque, sealed envelopes with a carbon copy paper. The serially numbered opaque envelopes will be packaged and prepared by an independent member not belonging to the research team. Participants will be randomised by the trial coordinator who will ensure that the envelopes are opened sequentially, and only after the participant's unique study identifier is written on the outside of the appropriate envelope.

\section{Blinding}

It is not possible to blind the study participants or the nurse delivering the intervention to the group allocations. However, study personnel involved in outcome assessment and data analysis will be blinded. Participants will be requested not to disclose group allocation to the outcome assessor, but if this does occur it will be recorded. Only once data have been cleaned and analysed will the treatment allocation be made known.

\section{Intervention}

The intervention will be delivered by the same nurse as in phase 1 , any additional nurses recruited will undergo the same training.

\section{Non-pharmacological component}

The non-pharmacological component will be delivered by a nurse as detailed in table 1 incorporating any modifications made following the development phase (a detailed description is included in online supplementary additional file 2). It will be delivered in up to six faceto-face sessions over 13 weeks. Participants will continue their usual analgesics in this period.

\section{Pharmacological component}

The nurse will take a history of current symptoms, comorbidities, medications and the main knee complaint of the participant. They will be advised by the nurse to continue on their current analgesia prescribed by their GP, and to add in simpler and safer analgesics in the sequence shown in figure 2. Once they are on a simpler and safer analgesic, they will be advised to reduce the dose of potentially more toxic or stronger analgesic. However, this will depend on the subjective improvement the participant experiences with simpler analgesic.

Relative and absolute contraindications will be assessed against a checklist developed from the British National
Formulary. Participants with absolute contraindication to an analgesic will not be prescribed that drug. Participants with relative contraindications may be prescribed the aforementioned drug if they are willing to do so after exploring risks and benefits.

Analgesics will be reviewed at two weekly intervals over a 13-week period and optimised if the pain relief is insufficient. This will be done over the phone, or at a face-to-face visit, depending on participant preference. The visits and telephone consultations will be conducted by the nurse and prescriptions signed by the principal investigator or nominated deputy. Once a participant achieves adequate pain control and does not request any further changes to their analgesia, they will be advised to contact the nurse by phone for changes to their treatment if needed during the study.

\section{Usual care}

Participants allocated to this group will continue to receive usual care for their knee pain. They will not undertake any of the clinical assessments and will not receive any input from the nurse. People in this group will be part of the baseline cohort and will not be aware of the content of the invention groups.

\section{Concomitant treatments}

Participation in the trial does not preclude the participants from receiving any concomitant care or treatment.

\section{Quantitative study outcomes}

The feasibility of running a full trial will be assessed by recording the following data:

- Recruitment rates.

- Dropout rate and reasons for dropout.

- Number of scheduled nurse appointments attended.

- Number of instances of unblinding.

- Completeness of questionnaire data.

- Concordance with exercise assessed using data from participants' exercise diaries (total number of days on which exercises were performed).

\section{Participant-reported measures}

A summary of all participant outcomes to be collected at 0,13 and 26 weeks for groups A and B is presented in table 2 and in online supplementary additional file 3 .

Group $\mathrm{C}$ will receive a questionnaire at week 26, enquiring about healthcare utilisation, demographic characteristics, self-reported height, self-reported weight, current alcohol intake and smoking status, comorbidities, medications, joint pain, central aspects of pain in knee scale, Western Ontario and McMaster Universities Osteoarthritis Index (WOMAC), Short Form-36 Health Survey version 2 and Hospital Anxiety and Depression Scale.

\section{Safety and adverse events}

This study intervention follows the current NICE guidelines that might be offered as part of routine clinical care. As such the risk of severe or unexpected adverse 
Table 1 Content of non-pharmacological component of intervention

\begin{tabular}{|c|c|c|c|c|c|c|}
\hline \multirow[b]{2}{*}{ Content of non-pharmacological component } & \multicolumn{6}{|c|}{ Session } \\
\hline & 1 & 2 & 3 & 4 & 5 & 6 \\
\hline \multicolumn{7}{|l|}{ Assessment } \\
\hline $\begin{array}{l}\text { Holistic assessment including symptoms, pain elsewhere, comorbidities, impact } \\
\text { on function, occupation, mood, sleep, illness perceptions, current levels of PA and } \\
\text { attitudes to PA and weight loss (if required) }\end{array}$ & $\checkmark$ & & & & & \\
\hline $\begin{array}{l}\text { Physical assessment of knee range of movement, lower limb muscle strength, } \\
\text { observation of gait and functional activities, BMI }\end{array}$ & $\checkmark$ & & & & & \\
\hline
\end{tabular}

Education and advice

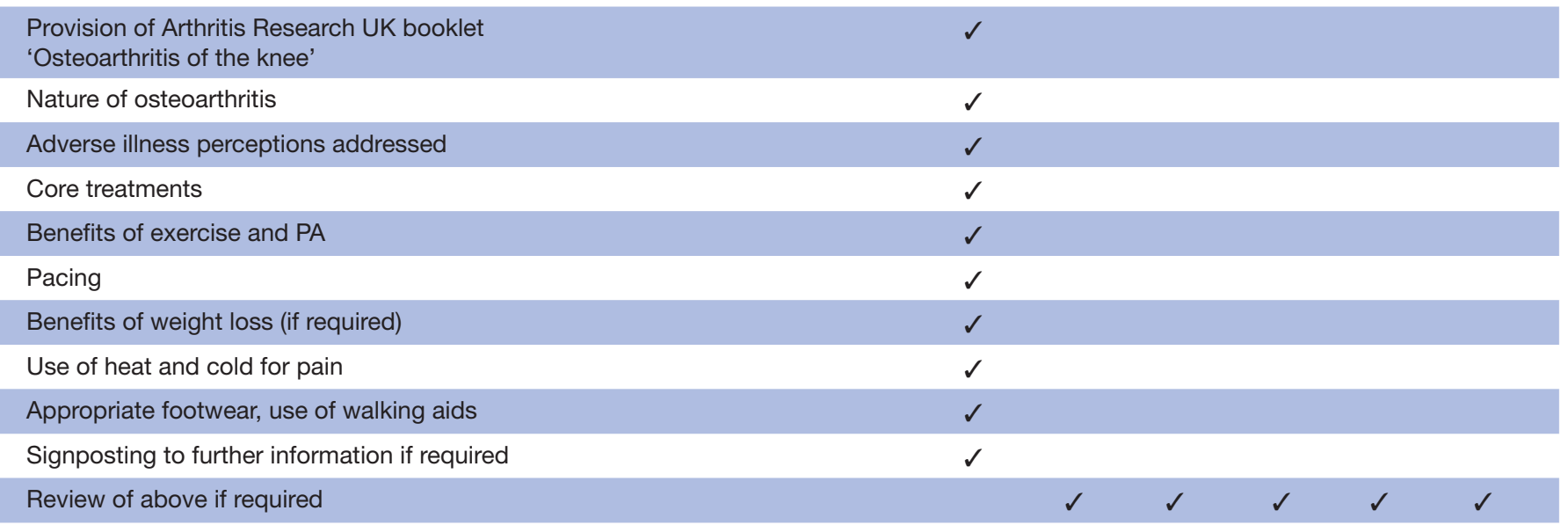

Exercise (individualised programme)

\begin{tabular}{|c|c|c|c|c|c|c|}
\hline Strengthening exercise & $\checkmark$ & & & & & \\
\hline Aerobic exercise/PA & $\checkmark$ & & & & & \\
\hline Functional exercises & $\checkmark$ & & & & & \\
\hline Review performance of exercise & & $\checkmark$ & $\checkmark$ & $\checkmark$ & $\checkmark$ & $\checkmark$ \\
\hline Progression/regression of exercises & & $\checkmark$ & $\checkmark$ & $\checkmark$ & $\checkmark$ & $\checkmark$ \\
\hline Previous efforts to lose weight discussed & $\mathcal{J}$ & & & & & \\
\hline Strategies for weight loss discussed & $\checkmark$ & & & & & \\
\hline Agree weight loss goal (5\% body weight) & $\checkmark$ & & & & & \\
\hline Signposting to resources (weight loss groups, NHS weight loss plan) & $\checkmark$ & & & & & \\
\hline Review of weight loss progress and advice & & $\checkmark$ & $\checkmark$ & $\checkmark$ & $\checkmark$ & $\checkmark$ \\
\hline Patient's confidence to achieve goals discussed and recorded & $\checkmark$ & $\checkmark$ & $\checkmark$ & $\checkmark$ & $\checkmark$ & $\checkmark$ \\
\hline Barriers and facilitators discussed & $\checkmark$ & $\checkmark$ & $\checkmark$ & $\checkmark$ & $\checkmark$ & $\checkmark$ \\
\hline Exercise diary completed and discussed & $\checkmark$ & $\checkmark$ & $\checkmark$ & $\checkmark$ & $\checkmark$ & $\checkmark$ \\
\hline
\end{tabular}

BMI, body mass index; NHS, National Health Service; PA, physical activity.

events (AE) is low. ${ }^{28}$ Exercise and an increase in physical activity may initially increase the risk of AEs such as pain, fatigue or muscle soreness or increased falls through increased activity. To reduce the risk of AEs the exercise programme will be tailored to the abilities of the participants. All serious and non-serious AEs will be monitored and recorded through the study by the nurses and will be managed in line with current National Institute for Health Research guidelines. ${ }^{29}$

\section{Qualitative study outcomes}

Acceptability of the intervention will be assessed using qualitative interviews conducted after the intervention (week 26) with approximately 10 participants 


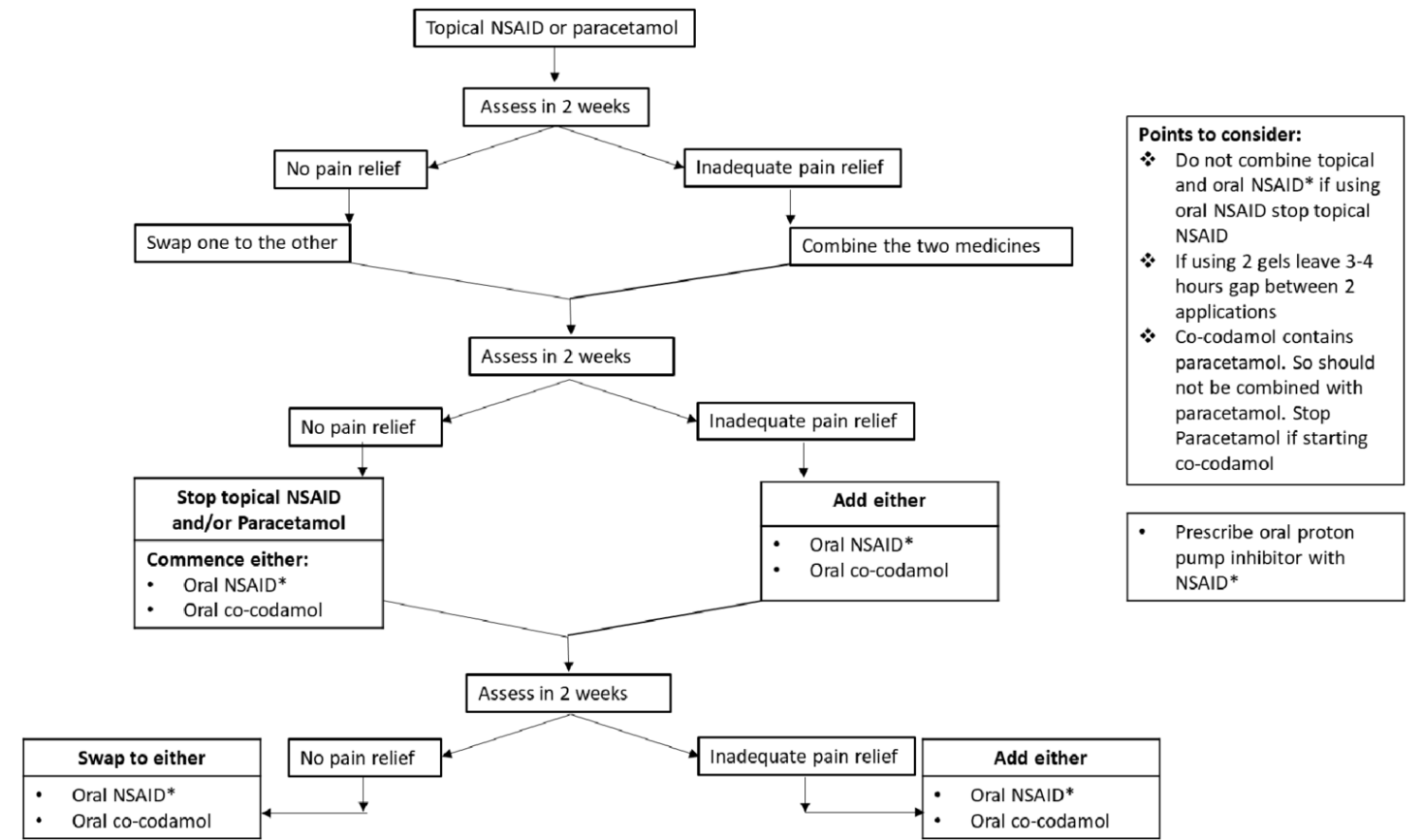

Figure 2 Analgesic sequence for pharmacological component. NSAID, non-steroidal anti-inflammatory drug.

from each of the intervention arms (groups A and B). They will be purposively selected to represent those with likely low and high concordance with the exercise advice using the Adherence to Exercise Scale for Older Patients questionnaire. Interviews will explore participants' overall satisfaction with the intervention and the sequence of treatment, perceptions of nurse-led care and previous treatment experience, level of adherence to the advice, perceptions of managing their knee pain, as well as perceived impact of their knee pain on their daily life before and after the intervention. Participants who withdraw from the intervention will be offered the opportunity to take part in an interview. Interviews will also be conducted with the study nurses to explore their experience in delivering the intervention, perceived effectiveness of the intervention and barriers to implementation and how these may be overcome.

\section{Sample size}

Quantitative study

As this is a feasibility study, a formal sample size calculation for between-group comparisons of primary clinical outcome is not appropriate. A target sample size of 53 participants per arm will be sought over the recruitment period to reliably estimate the feasibility outcomes relating to recruitment and retention rates to inform a fully powered RCT. With a sample size of 159 (53 participants per arm), we will be able to estimate a dropout rate of no more than $20 \%$ to within $7 \%$ points of the true value with $95 \%$ confidence.

\section{Qualitative study}

Target recruitment will be 20 participants, 10 from each intervention arm. However, final numbers will be determined by data saturation, where no new themes are identified.

\section{Data analysis}

Feasibility outcomes will be estimated using descriptive statistics (with 95\% CIs) and will be presented overall and per randomised groups. A Consolidated Standards of Reporting Trials (CONSORT) diagram will summarise the flow of participants through the study. Reasons for non-eligibility, withdrawals and non-completion of follow-up questionnaires will be presented if available. As this is a feasibility study, interim analyses are not planned. Similarly, missing data will not be imputed; on the contrary, the pattern of missing data will be assessed. Protocol non-adherence will be assessed as randomised.

\section{Quantitative analysis}

The main quantitative analysis will be of the trial feasibility outcomes:

- Recruitment rate (per month, per recruitment source, per 100 participants approached).

- Dropout rate (per arm, per stage pharmacological/ non-pharmacological).

- Attendance rates for scheduled nurse appointments.

- Missing data.

- Power and sample size calculation for a definitive trial will be based on WOMAC summated knee pain domain scores in the most painful knee at week 26.

Descriptive statistics will be presented for demographic data and all baseline clinical outcome measures. Exploratory analysis of clinical outcomes will be conducted according to randomised groups but will not be interpreted in terms of effectiveness. The emphasis will be on CIs of effect size estimations rather than the $p$ values. Changes 
Table 2 Summary of questionnaire and research measures to be collected

\begin{tabular}{|c|c|c|c|}
\hline Domain & Data source & Measure/instrument & $\begin{array}{l}\text { Time points } \\
\text { (week) }\end{array}$ \\
\hline $\begin{array}{l}\text { Demographic } \\
\text { characteristics }\end{array}$ & Research assessment & Age, height, weight & $\begin{array}{l}0 \\
0,13,26\end{array}$ \\
\hline $\begin{array}{l}\text { Radiographic } \\
\text { evaluation }\end{array}$ & Research assessment & $\begin{array}{l}\text { Bilateral knee radiographs: PA semiflexed weight bearing and skyline } \\
\text { views }{ }^{37} \text { scored for Kellgren and Lawrence grades and using the } \\
\text { Nottingham line drawing atlas }{ }^{38}\end{array}$ & 0 \\
\hline \multirow[t]{3}{*}{ Knee pain } & Self-report & $\begin{array}{l}\text { Western Ontario and McMaster Universities Osteoarthritis Index } \\
\text { (WOMAC) }^{39}\end{array}$ & $0,13,26$ \\
\hline & Self-report & Analgesic and NSAID consumption & $0,13,26$ \\
\hline & Research assessment & $\begin{array}{l}\text { Quantitative sensory testing* including pressure pain detection } \\
\text { threshold, temporal summation and conditioned pain modulation }\end{array}$ & $0,13,26$ \\
\hline Muscle function & Research assessment & Isometric and isokinetic quadriceps strength* & $0,13,26$ \\
\hline Quality of life & Self-report & $\begin{array}{l}\text { The Short Form-36 Health Survey V2 } \\
\text { EQ-5D-5L } \\
44\end{array}$ & $0,13,26$ \\
\hline $\begin{array}{l}\text { Psychological } \\
\text { wellness }\end{array}$ & Self-report & Hospital Anxiety and Depression Scale (HADS) ${ }^{45}$ & $0,13,26$ \\
\hline Healthcare use & Self-report & $\begin{array}{l}\text { Service use questionnaire to assess use of NHS or private healthcare, } \\
\text { prescription and over-the-counter medicines related to knee pain } \\
\text { outside of the study }\end{array}$ & $0,13,26$ \\
\hline $\begin{array}{l}\text { Participant } \\
\text { engagement in } \\
\text { treatment }\end{array}$ & $\begin{array}{l}\text { Nurse-completed } \\
\text { questionnaire }\end{array}$ & Pittsburgh Rehabilitation Participation Scale (PRPS) ${ }^{46}$ & 13/26† \\
\hline
\end{tabular}

*Further description included in detail in online supplementary additional files 3 and 4.

†This will be completed by the nurse after each of the non-pharmacological sessions.

$\ddagger$ This will be completed by the participant at the end of non-pharmacological arm.

§Online supplementary additional file 4.

EQ-5D-5L, 5-level version of EuroQol-5 Dimension; NHS, National Health Service; NSAID, non-steroidal anti-inflammatory drug; PA, physical activity.

in clinical and patient-reported outcomes from baseline to 13 and 26 weeks will be analysed using appropriate parametric or non-parametric statistics. A comparison of those receiving the pharmacological component first with those receiving the non-pharmacological component first will help determine the order of delivery in a future trial.

\section{Qualitative analysis}

Interview data will be analysed following the framework approach as described in phase $1 .^{30}$ Analysis will be conducted in parallel with the interviews and initial results will inform subsequent sampling and areas of interest to follow-up.

\section{CRITERIA FOR TERMINATING THE STUDY}

The study may be stopped by the sponsor if there is apparent futility in continuing with it.

\section{DATA MANAGEMENT}

Study data will be managed by the study coordinator (BM) under the supervision of the chief investigator (AA) and the study statistician (RO). A data monitoring committee has not been convened by the sponsor as this is a feasibility study using well-established and NICEapproved interventions. All trial staff and investigators will endeavour to protect the rights of the trial's participants to privacy and informed consent, and will adhere to the Data Protection Act 2018 and the General Data Protection Regulation (European Union) 2016/679. The study coordinator shall carry out monitoring of trial data as an ongoing activity. Trial data and evidence of monitoring and systems audit will be made available for inspection as required by the sponsor and the Research Ethics Committee (REC). 
Qualitative data and video or audio recordings will be archived in the University of Nottingham servers using password protection. Quantitative trial data will be stored in Research Electronic Data Capture database with range checks for data values. All quantitative data will be source data verified. Ten per cent of the entered trial data will be audited and variables for which there is $>5 \%$ error will be entered again. Once auditing is complete the electronic case report forms will be signed off and the database placed under hard lock. Study data will be available to the study statistician, $\mathrm{PhD}$ student and research fellow working on the project.

In compliance with the International Conference on Harmonization/Good Clinical Practice guidelines, regulations and in accordance with the University of Nottingham Research Code of Conduct and Research Ethics, the chief or local principal investigator will maintain all records and documents regarding the conduct of the study. These will be retained for at least 7 years or longer if required.

\section{ROLE OF SPONSOR AND FUNDERS}

There is no role of the study sponsors and funders in the design; collection, management, analysis and interpretation of data; writing of the report; and the decision to submit the report for publication. The study sponsor and funder will have no ultimate authority over any of these activities.

\section{ETHICS AND DISSEMINATION}

This protocol was given approval approved by the East Midlands-Derby REC (18/EM/0288) prior to commencing recruitment in November 2018 which is ongoing. The study was registered at ClinicalTrials.gov. The trial will be reported according to CONSORT guidance and standards. ${ }^{31}$ The results will be submitted for publication in peer-reviewed academic journals. Any modification to the approved protocol will result in resubmission to gain approval from the REC and study sponsor. Authorship eligibility guidelines will be adhered to, and we do not intend to use professional writers. Any interested researcher will be able to access the full protocol, participant-level data set and statistical code for research purposes after data sharing agreement has been signed.

\section{DISCUSSION}

People with knee OA continue to often receive suboptimal fragmented care and the core NICE recommendations are underused in primary care. ${ }^{32}$ Given that knee pain is common, and there is a huge time pressure on GPs in the $\mathrm{UK}$, it is vital to find out if a complex package of care incorporating the core recommendations can be delivered by other healthcare professionals such as practice nurses.

We believe that a wholly nurse-led management programme where a nurse acts as the point of contact for people with knee pain due to OA, educates them about the condition, provides core pharmacological and non-pharmacological treatments and builds a long-term therapeutic relationship is likely to be clinically effective and cost-effective in improving quality of $\mathrm{OA}$ care as demonstrated for other chronic conditions. ${ }^{1633-36}$

This study will not provide an answer as to the effectiveness of a wholly nurse-led intervention for $\mathrm{OA}$ and knee pain, but will determine the feasibility of implementing this model of care and of testing it in a full trial using practice nurses. Assessing fidelity will play an important part in the development and evaluation of the intervention, by exploring the extent to which the nurses can deliver individual components of the complex package of care for knee pain as planned. Combined with qualitative interviews to explore their acceptability, this will provide insight into the suitability of the training and intervention to inform appropriate refinements to the whole package of care that can be tested in a full trial. Although the intervention will take place in a research setting rather than primary care, the study outcomes will provide insight into the feasibility of implementation into real-world practice.

Further, this study will explore at what point analgesia should be optimised within this complex package of care. Having two intervention groups, one where analgesia is provided before the non-pharmacological component and one where it is provided after, will help us determine whether patients exercise better when analgesia is optimised first, or whether they are able to exercise sufficiently before this. This will inform the order of treatment in a two-arm full trial.

\section{Author affiliations}

${ }^{1}$ School of Health Sciences, University of Nottingham, Nottingham, UK ${ }^{2}$ NIHR Nottingham Biomedical Research Centre, University of Nottingham, Nottingham, UK

${ }^{3}$ Academic Rheumatology, University of Nottingham, Nottingham, UK ${ }^{4}$ Pain Centre Versus Arthritis, University of Nottingham, Nottingham, UK ${ }^{5}$ Nottingham Clinical Trials Unit, University of Nottingham, Nottingham, UK ${ }^{6}$ School of Medical and Surgical Sciences, University of Nottingham, Nottingham, UK ${ }^{7}$ Institute of Mental Health, University of Nottingham, Nottingham, UK

${ }^{8}$ Division of Psychiatry and Applied Psychology, University of Nottingham, Nottingham, UK

\section{Twitter Bonnie Millar @III_millar}

Contributors MH, PAN, BM, RO, AV, PG, RdN, MD, DAW and AA conceived the study. All authors contributed to the study design. MH, AF and AA wrote the manuscript. All authors critically revised the manuscript and approved the final version.

Funding This work was supported and cofunded by the NIHR Nottingham Biomedical Research Centre and the Pain Centre Versus Arthritis. The study is sponsored by the University of Nottingham, UK.

Disclaimer The views expressed are those of the author(s) and not necessarily those of the NHS, the NIHR or the Department of Health and Social Care.

\section{Competing interests None declared.}

Patient and public involvement Patients and/or the public were involved in the design, or conduct, or reporting, or dissemination plans of this research. Refer to the Methods section for further details.

Patient consent for publication Not required.

Provenance and peer review Not commissioned; externally peer reviewed.

Open access This is an open access article distributed in accordance with the Creative Commons Attribution Non Commercial (CC BY-NC 4.0) license, which permits others to distribute, remix, adapt, build upon this work noncommercially, and license their derivative works on different terms, provided the original work is properly cited, appropriate credit is given, any changes made indicated, and the use is non-commercial. See: http://creativecommons.org/ licenses/by-nc/4.0/. 
ORCID iD

Michelle Hall http://orcid.org/0000-0003-2231-1507

\section{REFERENCES}

1 Peat G, McCarney R, Croft P. Knee pain and osteoarthritis in older adults: a review of community burden and current use of primary health care. Ann Rheum Dis 2001;60:91-7.

2 Cross M, Smith E, Hoy D, et al. The global burden of hip and knee osteoarthritis: estimates from the global burden of disease 2010 study. Ann Rheum Dis 2014;73:1323-30.

3 Jinks C, Jordan K, Croft P. Osteoarthritis as a public health problem: the impact of developing knee pain on physical function in adults living in the community: (KNEST 3). Rheumatology 2007;46:877-81.

4 Grotle M, Hagen KB, Natvig B, et al. Prevalence and burden of osteoarthritis: results from a population survey in Norway. $J$ Rheumatol 2008;35:677-84.

5 NICE. Osteoarthritis: care and management in adults. London: National Institute for Health and Clinical Excellence, 2014.

6 Porcheret M, Jordan K, Jinks C, et al. Primary care treatment of knee pain--a survey in older adults. Rheumatology 2007;46:1694-700.

7 Ashaye T, Hounsome N, Carnes D, et al. Opioid prescribing for chronic musculoskeletal pain in UK primary care: results from a cohort analysis of the COPERS trial. BMJ Open 2018;8:e019491.

8 Conaghan PG, Porcheret M, Kingsbury SR, et al. Impact and therapy of osteoarthritis: the arthritis care oa nation 2012 survey. Clin Rheumatol 2015;34:1581-8.

9 Trouvin A-P, Berenbaum F, Perrot S. The opioid epidemic: helping rheumatologists prevent a crisis. RMD Open 2019;5:e001029.

10 van Tunen JAC, van der Leeden M, Bos WH, et al. Optimization of analgesics for greater exercise therapy participation among patients with knee osteoarthritis and severe pain: a feasibility study. Arthritis Care Res 2016;68:332-40.

11 Dziedzic KS, Healey EL, Porcheret M, et al. Implementing core NICE guidelines for osteoarthritis in primary care with a model consultation (mosaics): a cluster randomised controlled trial. Osteoarthritis Cartilage 2018;26:43-53.

12 Hay EM, Foster NE, Thomas E, et al. Effectiveness of community physiotherapy and enhanced pharmacy review for knee pain in people aged over 55 presenting to primary care: pragmatic randomised trial. BMJ 2006;333:995.

13 Dziedzic KS, Healey EL, Main CJ. Implementing the NICE osteoarthritis guidelines in primary care: a role for practice nurses. Musculoskeletal Care 2013;11:1-2.

14 Saffi MAL, Polanczyk CA, Rabelo-Silva ER. Lifestyle interventions reduce cardiovascular risk in patients with coronary artery disease: a randomized clinical trial. Eur J Cardiovasc Nurs 2014;13:436-43.

15 Strömberg A, Mårtensson J, Fridlund B, et al. Nurse-Led heart failure clinics improve survival and self-care behaviour in patients with heart failure: results from a prospective, randomised trial. Eur Heart $J$ 2003;24:1014-23.

16 Welch G, Garb J, Zagarins S, et al. Nurse diabetes case management interventions and blood glucose control: results of a meta-analysis. Diabetes Res Clin Pract 2010;88:1-6.

17 Primary Care Workforce Commission. The future of primary care: creating teams for tomorrow, 2015. Available: http://hee.nhs.uk/wpcontent/blogs.dir/321/files/2015/07/The-future-ofprimary-care.pdf

18 O'Reilly SC, Muir KR, Doherty M. Effectiveness of home exercise on pain and disability from osteoarthritis of the knee: a randomised controlled trial. Ann Rheum Dis 1999;58:15-19.

19 van der Velden JM, Verkooijen HM, Young-Afat DA, et al. The cohort multiple randomized controlled trial design: a valid and efficient alternative to pragmatic trials? Int J Epidemiol 2016;46:96-102.

20 Campbell R, Peters T, Grant C, et al. Adapting the randomized consent (Zelen) design for trials of behavioural interventions for chronic disease: feasibility study. J Health Serv Res Policy 2005;10:220-5.

21 Millar B, McWilliams DF, Abhishek A, et al. Investigating musculoskeletal health and wellbeing; a cohort study protocol. BMC Musculoskelet Disord 2020;21:1-10.

22 Moore GF, Audrey S, Barker M, et al. Process evaluation of complex interventions: medical Research Council guidance. BMJ 2015;350:h1258.

23 R S, L K, A J. The absent health professional: the educational needs of nurses, allied health professionals and associate practitioners working with people with osteoarthritis or rheumatoid arthritis: arthritis research UK 2013.

24 Dennick R. Twelve tips for incorporating educational theory into teaching practices. Med Teach 2012;34:618-24.

25 Toomey E, Matthews J, Hurley DA. Using mixed methods to assess fidelity of delivery and its influencing factors in a complex selfmanagement intervention for people with osteoarthritis and low back pain. BMJ Open 2017;7:e015452.

26 Ritchie J, Spencer L, O'Connor W. Carrying out qualitative analysis. qualitative research practice: a guide for social science students and researchers 2003;2003:219-62.

27 Srivastava A, Thomson SB. Framework analysis: a qualitative methodology for applied policy research 2009.

28 Niemeijer A, Lund H, Stafne SN, et al. Adverse events of exercise therapy in randomised controlled trials: a systematic review and meta-analysis. Br J Sports Med 2019:bjsports-2018-100461.

29 NIHR. Good clinical practice (GCP): reference guideV3.2: National Institute for health research 2017.

30 Gale NK, Heath G, Cameron E, et al. Using the framework method for the analysis of qualitative data in multi-disciplinary health research. BMC Med Res Methodol 2013;13:117.

31 Schulz KF, Altman DG, Moher D, et al. Consort 2010 statement: updated guidelines for reporting parallel group randomised trials. BMC Med 2010;8:18.

32 Østerås N, Jordan KP, Clausen B, et al. Self-Reported quality care for knee osteoarthritis: comparisons across Denmark, Norway, Portugal and the UK. RMD Open 2015;1:e000136.

33 Martínez-González NA, Tandjung R, Djalali S, et al. The impact of physician-nurse task shifting in primary care on the course of disease: a systematic review. Hum Resour Health 2015;13:55.

34 Driscoll A, Currey J, Tonkin A, et al. Nurse-Led titration of angiotensin converting enzyme inhibitors, beta-adrenergic blocking agents, and angiotensin receptor blockers for people with heart failure with reduced ejection fraction. Cochrane Database Syst Rev 2015;12:Cd009889.

35 Hendriks JML, de Wit R, Crijns HJGM, et al. Nurse-Led care vs. usual care for patients with atrial fibrillation: results of a randomized trial of integrated chronic care vs. routine clinical care in ambulatory patients with atrial fibrillation. Eur Heart J 2012;33:2692-9.

36 Denver EA, Barnard M, Woolfson RG, et al. Management of uncontrolled hypertension in a nurse-led clinic compared with conventional care for patients with type 2 diabetes. Diabetes Care 2003;26:2256-60.

37 Fernandes GS, Sarmanova A, Warner S, et al. Knee pain and related health in the community study (KPIC): a cohort study protocol. BMC Musculoskelet Disord 2017;18:404.

38 Nagaosa Y, Mateus M, Hassan B, et al. Development of a logically devised line drawing atlas for grading of knee osteoarthritis. Ann Rheum Dis 2000;59:587-95.

39 Bellamy N. WOMAC osteoarthritis index: a user's guide version 5. Brisbane 2002.

40 Hallal PC, Victora CG. Reliability and validity of the International physical activity questionnaire (IPAQ). Med Sci Sports Exerc 2004;36:556.

41 Mathias S, Nayak US, Isaacs B. Balance in elderly patients: the "getup and go" test. Arch Phys Med Rehabil 1986;67:387-9.

42 Dobson F, Hinman RS, Roos EM, et al. OARSI recommended performance-based tests to assess physical function in people diagnosed with hip or knee osteoarthritis. Osteoarthritis Cartilage 2013;21:1042-52.

43 Jenkinson C, Stewart-Brown S, Petersen S, et al. Assessment of the SF-36 version 2 in the United Kingdom. J Epidemiol Community Health 1999;53:46-50.

44 Rabin R, de Charro F, Fd C. EQ-5D: a measure of health status from the EuroQol group. Ann Med 2001;33:337-43.

45 Zigmond AS, Snaith RP. The hospital anxiety and depression scale. Acta Psychiatr Scand 1983;67:361-70.

46 Lenze EJ, Munin MC, Quear T, et al. The Pittsburgh rehabilitation participation scale: reliability and validity of a clinician-rated measure of participation in acute rehabilitation. Arch Phys Med Rehabil 2004;85:380-4.

47 Hardage J, Peel C, Morris D, et al. Adherence to exercise scale for older patients (AESOP): a measure for predicting exercise adherence in older adults after discharge from home health physical therapy. $J$ Geriatr Phys Ther 2007;30:69-78. 